Federal Reserve Bank of Minneapolis Quarterly Review Spring-Summer 1981
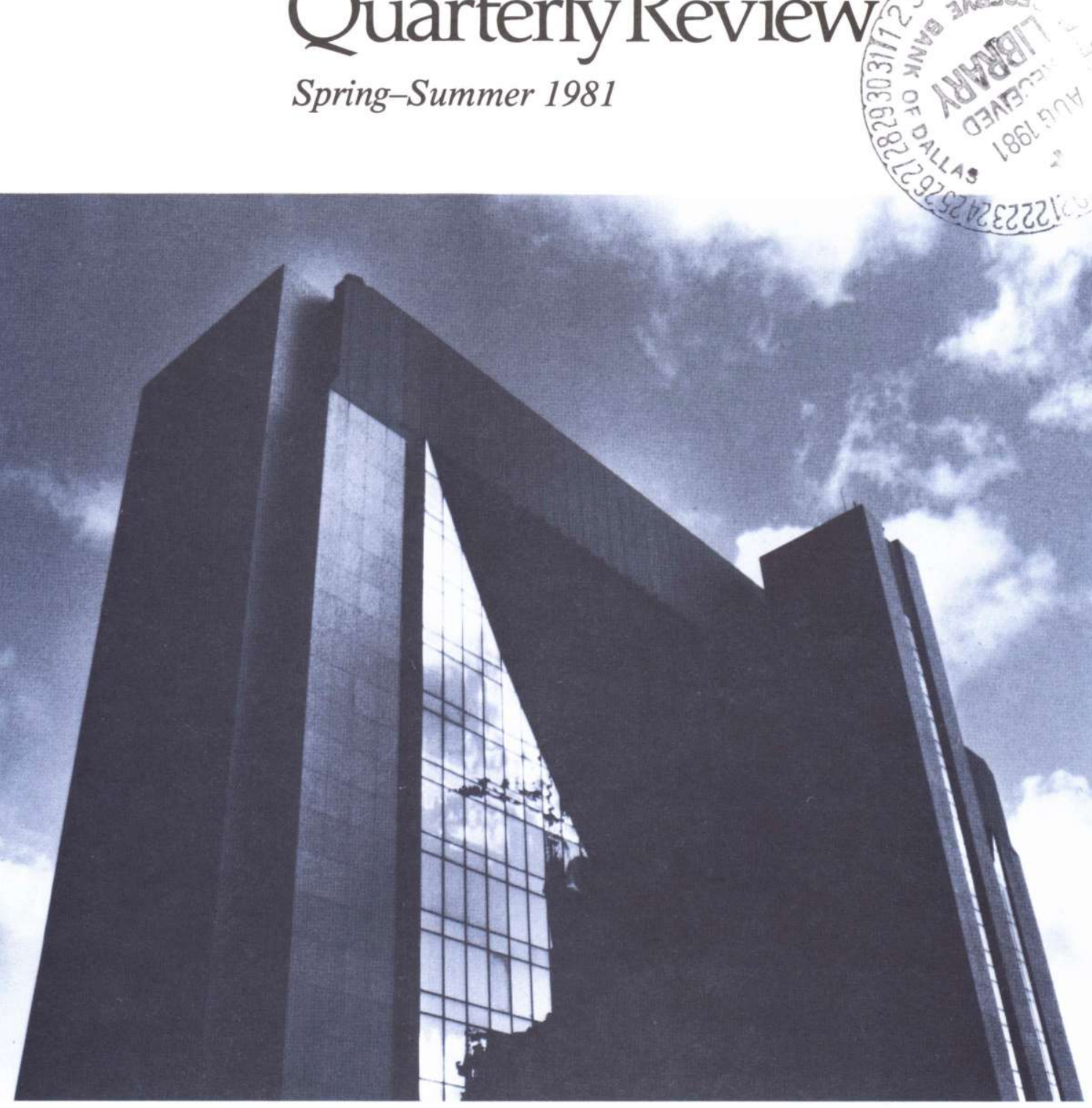
Federal Reserve Bank of Minneapolis

Quarterly Review vol. 5, No.2

This publication primarily presents economic research aimed at improving policymaking

by the Federal Reserve System and other governmental authorities.

Produced in the Research Department. Edited by Arthur J. Rolnick, Kathleen S. Rolfe, and Alan Struthers, Jr.

Graphic design and charts drawn by Phil Swenson, Graphic Services Department.

Address requests for additional copies to the Research Department,

Federal Reserve Bank, Minneapolis, Minnesota 55480.

Articles may be reprinted if the source is credited and the Research

Department is provided with copies of reprints.

The views expressed herein are those of the authors and not necessarily those of the Federal Reserve Bank of Minneapolis or the Federal Reserve System. 


\section{Econometric Policy Evaluation Under Rational Expectations}

\section{Thomas H. Turner, Economist}

Research Department

Federal Reserve Bank of Minneapolis

Charles H. Whiteman, Assistant Professor

University of Iowa

Econometric policy evaluation has had a dismal track record in recent years, as economists and policymakers have been notably unsuccessful in their attempts to project the consequences of unprecedented government policy changes. ${ }^{1} \mathrm{~A}$ theoretical explanation for these failures was advanced by Lucas (1976), who pointed out that the usage of traditional econometric models is flawed in a very serious way. In particular, standard techniques deny that economic agents change their behavior when the government changes its policy rules (repeated strategies for choosing policy variables). But if economic agents do alter their behavior in the subtle way Lucas described whenever government policies change, then techniques which ignore these responses cannot correctly project the results of policy changes.

Both recent practical experience and economic theory have thus seriously challenged the usefulness of econometric models as guides to evaluating alternative economic policies. This does not mean, however, that models are useless. ${ }^{2}$ It does mean that great care must be taken to evaluate alternative policies correctly.

The key to using econometric models for policy evaluation lies in correctly modeling the relationship between economic policy rules and descriptions of agents' patterns of behavior. This type of relationship is implied by the hypothesis that agents form expectations using all their information as best they can, that is, that they have $r a-$ tional expectations. Taking account of such relationships is hard to do in a large complicated econometric model and has yet to be accomplished on that scale. However, in what follows, we demonstrate how a very simple econometric model can be used to gauge both the expected effects of a new policy rule and the range of uncertainty surrounding the expected effects. We also show that more superficial, shortcut methods aimed at accounting for the effects of rational expectations can be as misleading as the discredited standard policy evaluation techniques they are meant to replace.

\section{The Right Way to Incorporate Rational Expectations}

Economists have long realized that economic agents' expectations, particularly about future government policy actions, impinge directly on their current decisions. Typically, economists have attempted to model this relationship in a very ad hoc way, by using an adaptive mechanism in which the expected value of a variable is determined from a distributed lag of past values of that variable itself. The rational expectations hypothesis implies a more sophisticated economic structure: the coefficients in equations describing agents' behavior (for example, coefficients of supply and demand curves) must be explicitly tied in very specific ways to the coefficients (or policy parameters) in equations describing economic policies. Economists refer to these (perhaps very complicated) ties between the coefficients in a behavioral equation and the policy parameters as cross-equation restrictions. That is, the coefficients of one equation in the

'For example, as Sargent (1980, p.16) points out, "Standard Keynesian and monetarist econometric models built in the late 1960 s failed to predict the effects on output, employment, and prices that were associated with the unprecedented large deficits and rates of money creation of the 1970s."

${ }^{2}$ See, for example, Lucas 1976, Sargent and Wallace 1975, Lucas and Sargent 1979, Anderson 1978 and 1979, Sargent 1980, and Supel 1980. 
model (the behavioral equation) must be restricted by the coefficients (policy parameters) of another equation (the policy rule) in the sense that the former must be made an explicit function of the latter.

The ability of the rational expectations hypothesis to deliver appropriate cross-equation restrictions for econometric policy evaluation is the feature that distinguishes rational expectations econometric models. We will demonstrate how this distinguishing feature works, using a very simple framework and explicit assumptions about both the information economic agents have and the way they use it to make forecasts.

The paradigm we will use is a simple two-equation model describing the demand for real money balances and a rule governing the money supply. This model and the forecasts generated from it will represent part of the information the monetary authority uses when choosing a monetary rule to effect some desired growth in the price level. The model is given in terms of the natural logarithms of the price level and the money supply, $p$ and $m$, respectively, in the relations below:

$$
\begin{aligned}
& m_{t}-p_{t}=-\left(E_{t} p_{t+1}-p_{t}\right)+v_{t} \\
& m_{t}=\rho m_{t-1}+e_{t} .
\end{aligned}
$$

Expression (1) is a version of Cagan's (1956) money demand equation relating the demand for real money balances to the expected rate of price inflation, that is, the difference between the price level expected at time $t$ to prevail at time $t+1, E_{t} p_{t+1}$, and the actual price level at time $t, p_{r}$. Expression (2) is a simple statement that the money supply at time $t$ is determined from the money supply at time $t-1$ through the value of $\rho$, the coefficient which indexes the monetary rule chosen by the monetary authority. ${ }^{3}$ The terms $v_{t}$ and $e_{t}$ are random variables accounting for the unexplained portions of $p_{t}$ and $m_{t}$, respectively. ${ }^{4}$

A policymaker attempting to use relations (1) and (2) for econometric policy evaluation needs to solve the model in order to derive an expression which can be used to predict future values of the price level. In order to do this, the policymaker must make some assumption about the way the expectation of next period's price level, $E_{t} p_{t+1}$, is formed. Here we assume this expectation is formed rationally.

Imposing rational expectations restricts the form of the solutions to the model in very particular ways, and it is these restrictions which permit the policymaker to use the model successfully for econometric policy evaluation. By imposing rationality, we are saying that agents' forecasts of future values of the price level are those which are produced according to relevant economic theory, equations (1) and (2) in our framework. The implication is that the monetary authority cannot systematically fool the public when it changes the monetary rule $\rho$, because agents know that the rule has changed and they alter their expectations of future prices accordingly. ${ }^{5}$

What this means, in terms of our model, is that the solution for the price level depends on both the money supply and the monetary rule $\rho$. This relationship turns out to be be $^{6}$

$$
p_{t}=\frac{1}{2-\rho} m_{t}-\frac{1}{2} v_{t}
$$

in which, clearly, the coefficient on current money $m$, is intimately tied to the monetary rule $\rho$ given in equation (2). That is, the coefficient $1 /(2-\rho)$ in $(3)$ is not free, but is restricted by the form of the rule described by (2).

By using (2) directly in (3), we can derive an alternative, but equivalent, form of the price equation which is more useful for forecasting because it gives the current price level $p_{t}$ in terms of the known money supply last period, $m_{t-1}$. This relation is

$$
p_{t}=\frac{\rho}{2-\rho} m_{t-1}+\frac{1}{2-\rho} e_{t}-\frac{1}{2} v_{t} .
$$

The complete solution of the model, (4) and (2), clearly displays the cross-equation restrictions resulting from the

${ }^{3}$ The rationality hypothesis requires that we focus on rules governing the policymaker's choices of economic policy variables as opposed to isolated actions taken by policymakers. In our demonstration here, references to $\rho$ as the monetary rule should be interpreted as a shorthand way of referring to the overall strategy or rule which defines the way policymakers choose alternative values of $\rho$. This rule is quite distinct from isolated, one-time actions which change the existing stock of money.

${ }^{4}$ We restrict the value of $\rho$ to be greater than zero. The disturbances $v_{t}$ and $e_{i}$ are each serially uncorrelated random variables with mean zero and are uncorrelated with all past values of the money supply.

${ }^{5}$ The rational expectation of next period's price level, $E_{t} p_{t+1}$, is the best linear projection of the price level based on the history of both prices and the money supply. In our discussion, we do not attempt to deal with the question of how fast agents catch on to the rule change; we merely assume that they do catch on, through observation or announcement, quickly.

${ }^{6}$ The technical details of the exercises described in this article are developed in Whiteman 1981 . 
rationality assumption. Agents' forecasts of future values of the price level will be generated using (4), where the coefficients are restricted by the monetary authority's choice of the monetary rule $\rho$. These are the correct restrictions in a world of rationality because they are derived explicitly from the monetary rule agents know about and use to form their expectations.

The evaluation of alternative monetary rules proceeds, in principle, according to the following scheme. First the policymaker assumes alternative values of $\rho$ and uses relation (2) to generate alternative sequences of values for the money supply $m$. Then, using these values for $m$ in (4), the policymaker generates alternative sequences of values for the one-period-ahead price level. Finally, a policymaker attempting to effect some desired average rate of price inflation over the next $T-t$ periods chooses the value of $\rho$ that produces a projected price sequence $p_{t+1}, \ldots, p_{T}$ in which the best prediction of the price level at time $T, p_{T}$, is $\bar{p}$, the target price level consistent with the desired average rate of inflation.

In our model, the forecast of $p_{T}$ will depend on the choice of the new rule-say, $\rho_{0}$ - as well as the current money supply $m_{t}$ and the forecast horizon $T-t$. Therefore, if the targeting problem is to try to make $p_{T}=\bar{p}$, the authority will choose that $\rho_{0}$ which equates the model's prediction for $p_{T}$ to the target price level, ${ }^{7}$ that is,

$$
\frac{\rho_{0}^{T-t} m_{t}}{2-\rho_{0}}=\bar{p} .
$$

An estimate of the ordinary uncertainty surrounding this prediction can be calculated from the properties of the residuals in (1) and (2), and this estimate can be used to construct a 70 percent confidence interval about the mean prediction $\bar{p}{ }^{8}$ The calculated confidence interval will also exhibit the restrictions imposed by rationality; that is, the confidence interval will depend on the value of $\rho_{0}$ chosen by the policymaker. Assuming that agents quickly catch on to the change from the historical $\rho$ to the new $\rho_{0}$, the likely range of the actual price level at time $T$ after the change in the monetary rule at time $t$ will be given by the prediction $\bar{p}$ and the associated 70 percent confidence interval: ${ }^{9}$

$$
\bar{p} \pm 1.04 \sqrt{\frac{1}{4}+\left(2-\rho_{0}\right)^{-2} \sum_{k=0}^{T-t-1} \rho_{0}^{2 k}} .
$$

To make our demonstration more concrete, suppose that both the money supply and the price level have been growing at an average rate of 20 percent per period over recent history, that is, that $\rho=1.2 .{ }^{10}$ To simplify the arithmetic, further assume that $m_{t}=1$. If the policymaker's goal were to reduce the average rate of inflation over the next $T-t$ periods to 10 percent per period using only the monetary rule $\rho$, the policymaker would try to find that value of $\rho$ for which the model's best prediction of the price level at time $T$ would be

$$
\bar{p}=(1.10)^{T-t} p_{t}
$$

Figure 1 shows the period-by-period projection of the price level based on the policy choice $\rho_{0}$ as derived from the above expression, as well as the 70 percent confidence interval associated with the model's best, or mean, prediction each period over the $T-t$ periods. ${ }^{11}$ If economic agents correctly perceive the rule change and if expectations are formed rationally, the restricted model can be successfully used to project the consequences of the rule change. Figure 1 then shows the best forecasting the policymaker could do; the projection correctly characterizes the true, or actual, evolution of prices (and the associated 70 percent confidence interval at each period) resulting from the policy change from the historical $\rho=1.2$ to the new $\rho_{0}$.

'It is easy to show that under rationality the reduced form prediction of the price level $j$ periods ahead is given by $\rho^{\prime} m_{i} /(2-\rho)$.

${ }^{8} \mathrm{~A} 70$ percent confidence interval is, in simplest terms, a range of values around a forecast in which the actual value of the projected variable will fall 70 percent of the time. The range of values is derived from the uncertainties in the policymaker's model.

${ }^{9}$ In deriving the confidence interval, we have computed the conditional variance from the vector moving average representation, dropping cross terms with zero expectation. We have also assumed that the variance-covariance matrix of the residual vector $(e, v)^{\prime}$ is the identity matrix, a harmless assumption for this illustrative example. The residual vector is assumed to be normally distributed.

Each confidence interval calculated and illustrated in this paper is actually a minimum estimate, since in fact the parameters of the reduced form are not known exactly, as we have assumed, but must be estimated. In practice, where the variance-covariance matrix of the residual vector is not known, the 70 percent confidence interval can be computed by drawing repeated realizations of the vector from a normal distribution, generating sequences of $p$ and $m$, and dropping the highest and lowest 15 percent values at each $t$.

${ }^{10}$ Strictly speaking, in our example it is the natural logarithms which have been growing at 20 percent per period.

"For all of the examples depicted here, the number of periods is $T-t=18$. Each time path shown has been detrended for scaling purposes by the reduced form projections of the correctly restricted model, $\left(2-\rho_{0}\right)^{-1} \rho_{0}^{\prime} m, j=1,2, \ldots, 18$. The variance-covariance matrix of the residual vector was scaled to provide stark contrasts; that is, the matrix $I$ was scaled by the factor 0.00002231 . 
Figure 1

Actual Evolution of Prices Under the Rational Policy

(Formulated With Cross-Equation Restrictions)

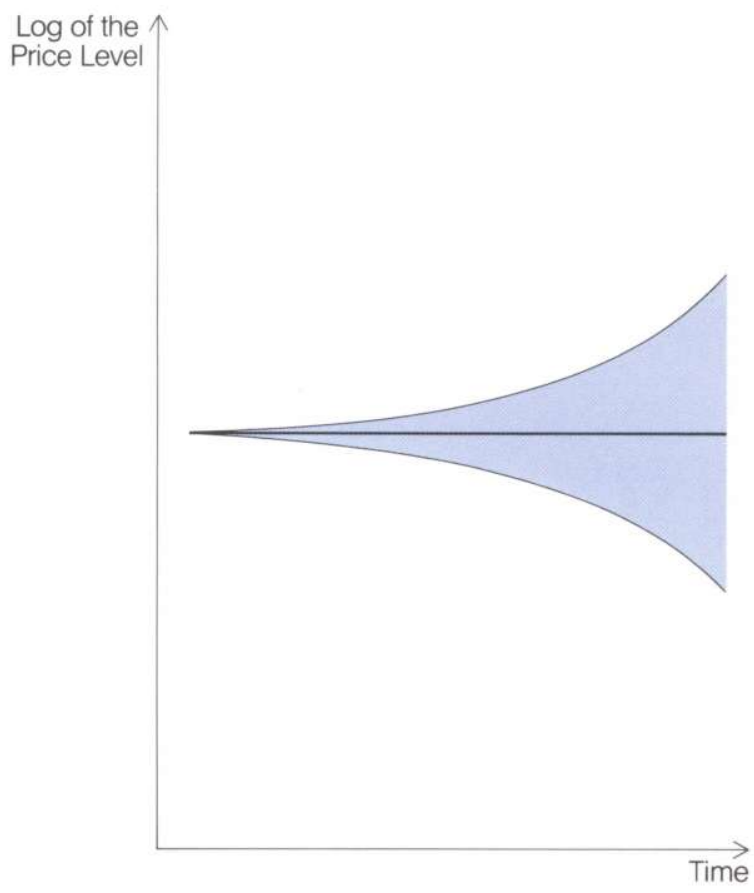

\section{Several Misleading Shortcuts}

The key to the successful policy evaluation exercise above lies in correctly imposing the cross-equation restrictions that rationality implies. Failure to capture these restrictions yields predictions and confidence intervals that lead to policy choices which result in consequences very different from those sought by the policymaker. It is exactly this failure which characterizes standard econometric policy evaluation.

Although we have shown in a very simple framework how the correctly imposed cross-equation restrictions derived from rational expectations can lead to projections which can be successfully used for policy evaluation, we have been careful to avoid suggesting that incorporating these restrictions into large econometric models is a trivial matter. Indeed, pioneering work in this area by Hansen and Sargent (1980) suggests that the task is anything but easy. Unfortunately, it is also true that so far no shortcuts to constructing successful econometric policy evaluation techniques have been developed.

Below, in the context of our simple model, we demonstrate why some of the shortcuts which have been proposed simply don't work. Briefly, the reason is that they do not correct the shortcoming of traditional methods, that is, the failure to account for the cross-equation restrictions between behavioral coefficients and policy parameters.

\section{A Shift From Point Predictions to Confidence Intervals}

Some have argued that the effect on econometric policy evaluation of omitting the correct cross-equation restrictions is likely to be small. ${ }^{12}$ Those who sympathize with this argument seem to think that the effects of alternative policies are likely to be captured in the confidence intervals calculated with traditional methods. This strategy is actually a reinterpretation, rather than a modification, of standard approaches to econometric modeling, a shift of emphasis from a model's point, or best, predictions to the confidence intervals around those predictions. However, as we demonstrate with our simple model, the errors from omitting the correct restrictions need not be small and both the point predictions and the confidence intervals resulting from the use of traditional techniques can be seriously misleading.

Note first that the policymaker using standard techniques has estimates of the money supply rule described by (2), just as we had in our example above. However, the practitioner of standard methods does not include agents' understanding of this rule in their expectations formation and thus estimates a relationship between the price level and the money supply which looks like this:

$$
p_{t}=\theta m_{t}-\frac{1}{2} v_{t}
$$

The error in using this relationship comes from the failure to recognize that the coefficient on current money, $\theta$, is in fact actually equal to $1 /(2-\rho)$, as in (3). In this standard framework, the price level and the money supply are assumed to be related through the (estimated) historical value of $\theta$, regardless of changes in the rule $\rho$.

By using the money supply rule (2) in (5), the policy-

\footnotetext{
${ }^{12}$ See, for example, the comments in Modigliani 1978 and in Solow 1978.
} 
maker generates future paths for the price level for alternative money sequences consistent with different values of $\rho$ according to the following relation:

$$
p_{t}=\theta \rho m_{t-1}+\theta e_{t}-\frac{1}{2} v_{t}
$$

Because $\theta$ is assumed invariant with respect to changes in $\rho$, the projections for the price level from (6) will obviously differ from those derived from (4) for particular choices of $\rho$. As a result, the policymaker solves the targeting problem, trying to make $p_{T}=\bar{p}$, by choosing a different monetary rule, $\rho_{1}$, which equates the prediction of $p_{T}$ from (6) with $\bar{p}$, that is,

$$
\theta \rho_{1}^{T-t} m_{t}=\bar{p} .
$$

The sequence of projections and associated 70 percent confidence intervals that the policymaker using standard techniques claims for the evolution of future price levels $p_{t+1}, p_{t+2}, \ldots, p_{T}$ is then given by

$$
\theta \rho_{1}^{j} m_{t} \pm 1.04 \sqrt{\frac{1}{4}+\theta^{2} \sum_{k=0}^{j-1} \rho_{1}^{2 k}}, j=1,2, \ldots, T-t .
$$

Use of the erroneous $\theta$ parameter obviously invalidates not only the projected future price levels, but also the confidence intervals associated with them.

Figure 2 shows the sequence of price level projections and confidence intervals claimed by the policymaker using standard techniques for the same problem which led to the sequence of projections shown in Figure 1. Clearly, the sequence shown in Figure 2 for the standard policy $\rho_{1}$ is not the same as that in Figure 1 for the rational expectations policy $\rho_{0}$ (chosen on the basis of the correctly restricted model). Additionally, the confidence intervals shown in Figure 2 cannot be interpreted as taking care of the uncertainty due to the policy change because, as the expression above makes clear, they also fail to reflect the policy change correctly.

Provided agents' expectations are rational, we may use the implied restrictions to show that the projected evolution of the price level claimed above for the policy $\rho_{1}$ is, in fact, not the actual evolution of the price level that would result from that policy choice. To see how the error comes
Figure 2

Claimed Evolution of Prices Under the Standard Policy

(Formulated Without Cross-Equation Restrictions)

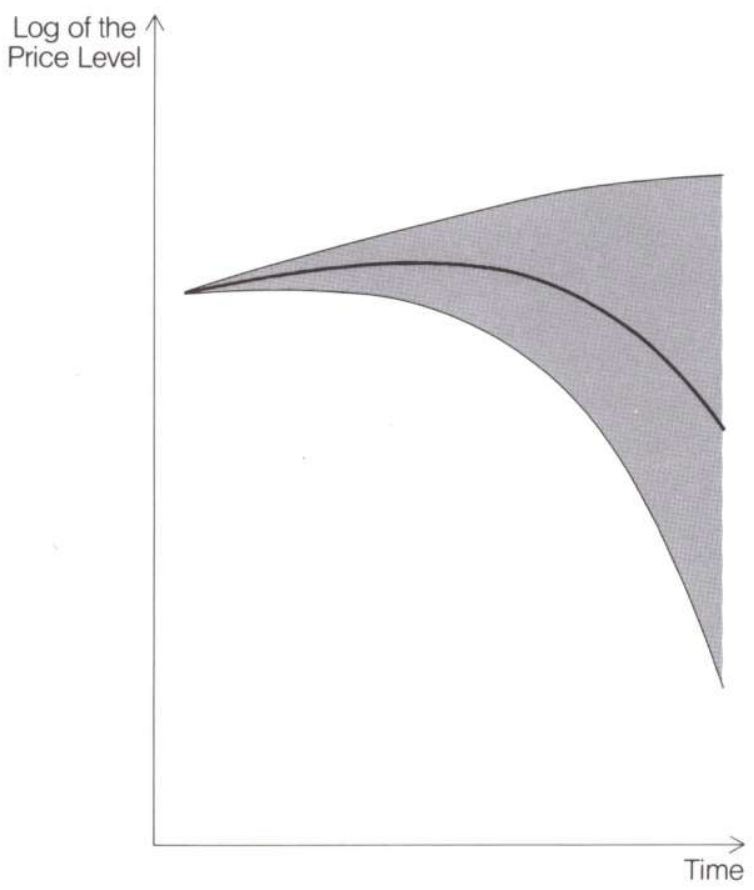

about, note that the projection of the price level at time $t+j$ claimed above for the policy $\rho_{1}$ is $\theta \rho_{1}^{j} m_{t}$, where $\theta$ is estimated over some historical period and is assumed to be invariant under the change to the new rule $\rho_{1}$. Imposing the correct cross-equation restrictions implied by rationality, however, showed that the best prediction of $p_{t+j}$ depends on the current money supply and on the new policy rule $\rho_{1}$ in the following way:

$$
\frac{\rho_{1}^{j} m_{t}}{2-\rho_{1}}
$$

Thus, under the policy $\rho_{1}$, the sequence of predictions and 70 percent confidence intervals that describes the actual evolution of future price levels $p_{t+1}, p_{t+2}, \ldots, p_{T}$ once agents 
realize that the new rule is in effect is

$$
\frac{\rho_{1}^{j} m_{t}}{2-\rho_{1}} \pm 1.04 \sqrt{\frac{1}{4}+\left(2-\rho_{1}\right)^{-2} \sum_{k=0}^{j-1} \rho_{1}^{2 k}}, j=1,2, \ldots, T-t .
$$

For the same problem used to generate Figures 1 and 2, Figure 3 contrasts the claimed evolution of prices and associated confidence intervals for the policy $\rho_{1}$ (from Figure 2) with the actual evolution of prices and confidence intervals for that policy. The contrast is a striking demonstration of the thrust of the Lucas critique. Under a policy rule chosen using standard techniques (ignoring cross-equation restrictions), the actual evolution of the price level can differ dramatically from that claimed by the policymaker using those techniques and the claimed confidence intervals can be both off-center and of incorrect size

\section{Figure 3}

Claimed and Actual Evolution of Prices Under the Standard Policy

(Formulated Without Cross-Equation Restrictions)

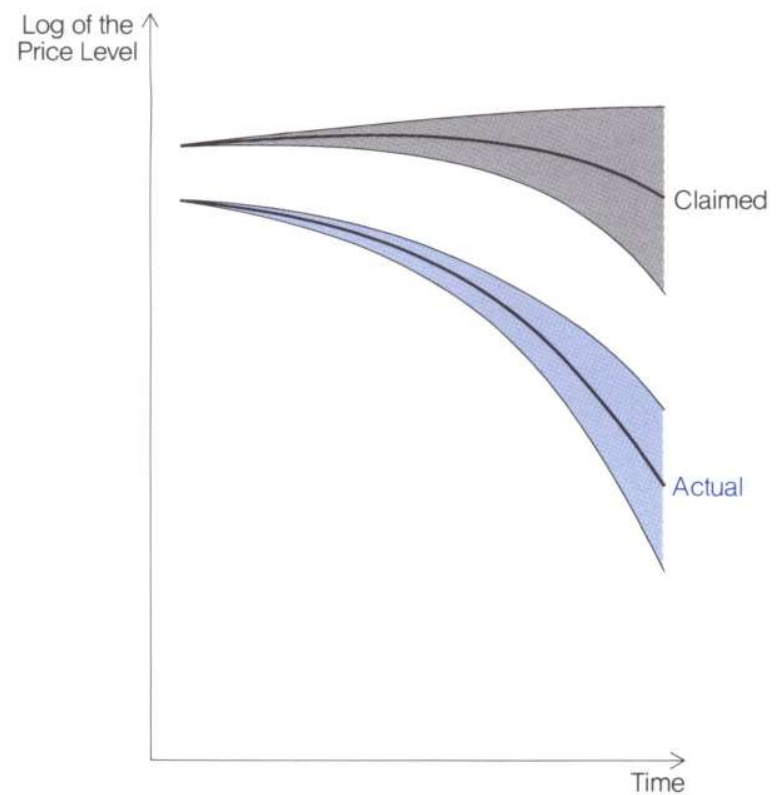

Figure 4

Actual Evolution of Prices Under the Rational and Standard Policies

(Formulated With and Without Cross-Equation Restrictions)

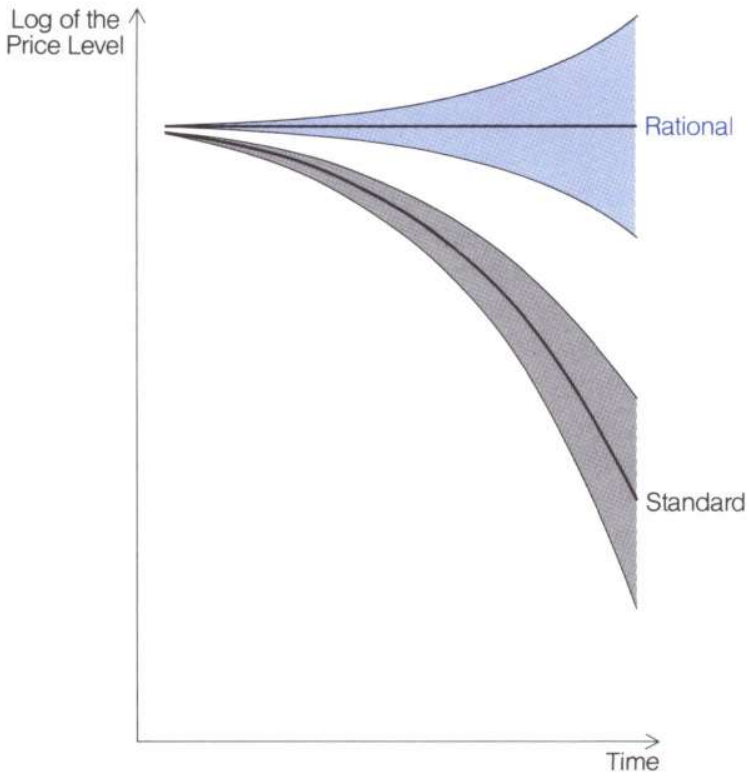

and shape. It is clear that for some values of the historical $\rho$ and the new $\rho_{1}$, the claimed and actual confidence intervals need not even overlap. It is also clear that the effect of omitting the cross-equation restrictions is neither necessarily small nor likely to be accounted for by appending standard confidence intervals to erroneous projections.

Figure 4 presents further evidence that policymakers should incorporate the correct restrictions into their forecasting models. The actual range of outcomes under the policy rule chosen when the correct restrictions are taken into account may differ significantly over the forecast horizon from the actual range of outcomes under the erroneous policy chosen when the correct restrictions are ignored. Clearly, no simple relation between the two paths need exist at all, and for this reason policymakers should not be surprised when standard techniques lead to results 
significantly different from those desired. ${ }^{13}$

\section{A Simulation Technique}

Another way of accounting for rational expectations in econometric models was suggested by Anderson (1978, 1979), who used a simulation method to equate a model's projection of the path of future prices to agents' expectations of that path. Anderson was trying to illustrate the types of effects that one might expect from policy rule changes that agents caught on to very quickly. Unfortunately, some investigators have mistakenly concluded that pursuing Anderson's simulation method-which we will call a pseudorational strategy-would provide a relatively easy shortcut to the results of correctly imposing the cross-equation restrictions. As we show below, this approach can give projections that are very different from the actual evolution of prices under rationality and therefore can imply policy rules that are very different from the correct ones to reach the policymaker's goals.

In practice, because of certain timing ambiguities which Anderson discusses, there are two choices about how to implement the pseudorational idea. ${ }^{14}$ The first choice is to use relation (5) to generate the expectation of next period's price level, $E_{t} p_{t+1}$, and to substitute this result into equation (1) to obtain

$$
p_{t}=\theta^{\prime} m_{t}-\frac{1}{2} v_{t}
$$

where $\theta^{\prime}=(1+\theta \rho) / 2$. However, now $\theta^{\prime}$ is assumed to be invariant under changes in the rule $\rho$, and therefore the same types of problems which invalidated the first shortcut method will invalidate this approach too.

In our framework, the second way to implement the pseudorational idea amounts to replacing $E_{t} p_{t+1}$ in equation (1) with the actual price level next period, $p_{t+1}$. Thus, (1) becomes

$$
m_{t}-p_{t}=-\left(p_{t+1}-p_{t}\right)+w_{t}
$$

where $w_{t}$ is the unexplained residual. Rearranging gives a solution for next period's price level as

$$
p_{t+1}=2 p_{t}-m_{t}+w_{t}
$$

in which it is clear that next period's price level, $p_{t+1}$, is known exactly in the current period $t .{ }^{15}$ The appropriate solution procedure is to begin with the target $p_{T}=\bar{p}$ and to solve backwards from time $T$ for the price and money sequences. The policy rule is found by determining the value of $\rho$-say, $\rho_{2}$-which equates the model's best prediction of $p_{T}$ to the objective $\bar{p}$, that is, the solution to ${ }^{16}$

$$
\bar{p}=2^{T-t} p_{t}-2^{T-t-1}\left[\frac{1-\left(\frac{\rho_{2}}{2}\right)^{T-t}}{1-\frac{\rho_{2}}{2}}\right] m_{t} .
$$

Clearly, this expression is very different from that derived when expectations are formed rationally and agents

\footnotetext{
${ }^{13}$ One approach to modifying standard techniques that we don't explicitly deal with here, but which might be viewed as a response to Lucas' critique, is to construct models as if the true coefficients were random in nature and to use this property in calculating forecasts and confidence intervals. An excellent example of this approach is described in Swamy and Tinsley 1980. In our framework, the standard technique is modified by characterizing $\theta$ and $\rho$ as having probability distributions and by updating the characterization of these coefficients for each period over which the model is estimated or used to predict. The information contained in this approach-about both the uncertainty surrounding the mean projections and the nature of the change in the coefficients and forecast error variability over time - could possibly be useful to policymakers.

However, on at least three grounds this strategy fails to salvage traditional methods of econometric policy evaluation. First, as Lucas emphasized in his critique, either randomness or drift observed in coefficients is merely a symptom of the basic failure to roll back econometric models to the levels where parameters do remain invariant, and this approach therefore fails to address the main thrust of the critique. Second, since the approach ignores the cross-equation restrictions, it does not appear to correct the mean, or best, conditional forecasts from standard techniques in ways which reflect the effects of policy changes. Thus, even if $\theta$ and $\rho$ in our model are characterized as truly random, the mean forecasts generated will not be the correct ones consistent with the cross-equation restrictions. Third, this approach fails to capture in a clear way the effects of a specific policy change, since it characterizes any definite structural change (such as a decision to change $\rho$ from its historical value to the new $\rho_{1}$ ) as one that with only some (perhaps small) probability may occur.

${ }^{14}$ See the discussion in Anderson 1979, pp. 71-73.

${ }^{15}$ In our framework, we can derive an explicit solution which is equivalent to the results that would be obtained from model simulations.

${ }^{16}$ In the solution presented, $\rho$ is constrained to be less than 2 in absolute value. The forecast and associated 70 percent confidence interval for the price level $j$ periods from time $t$ are given by
}

$$
\begin{aligned}
& 2^{j} p_{t}-2^{j-1}\left[\frac{1-\left(\frac{\rho}{2}\right)^{j}}{1-\frac{\rho}{2}}\right]_{t}+2^{j-1} v_{t} \\
& \pm 1.04 \sqrt{2^{2(j-1)} \sum_{r=1}^{j-1}\left(\sum_{k=1}^{j-1}\left(\frac{1}{2}\right)^{k} \rho^{k-t}\right)^{2} \sigma_{r}^{2}+\frac{1}{3}\left(2^{2(j-1)}\right) \sigma_{v}^{2}}
\end{aligned}
$$

where $\sigma_{e}^{2}$ and $\sigma_{x}^{2}$ are the variances of the disturbance terms $e$ and $v$, respectively. 
Figures 5 and 6

\section{Evolution of Prices Under the Pseudorational Policy}

Figure 5

Claimed

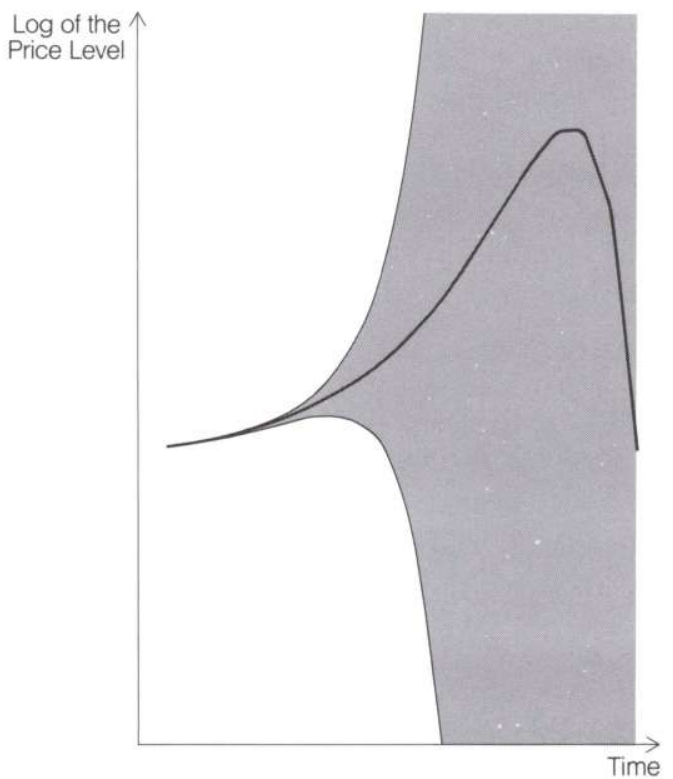

Figure 6

Actual

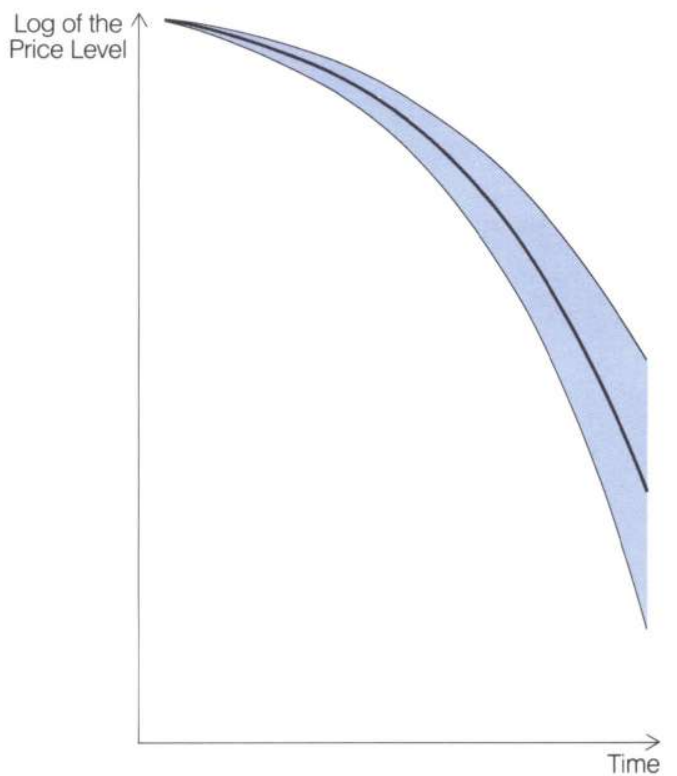

catch on to monetary rule changes quickly. That is, the pseudorational approach does not deliver, in our model, anything that even vaguely resembles the solution from correctly imposing the cross-equation restrictions.

To demonstrate the results of this strategy, we have used the same problem posed earlier. Figure 5 shows the claimed evolution of the price level using the rule implied by the pseudorational strategy. Not only does the price sequence differ dramatically from that projected in the correctly restricted case (see Figure 1), but the 70 percent confidence interval essentially explodes. This happens even though we have been charitable by assuming that the forecaster correctly knows that the coefficient on the expected price level, $E_{t} p_{t+1}$, is -1 . In fact, as Anderson observes, this coefficient is typically estimated incorrectly under ad hoc assumptions about the way expectations are modeled. ${ }^{17}$

Just as we demonstrated earlier for prices under the policy choice $\rho_{1}$ made using standard techniques, we can show that the claimed evolution of the price level under the pseudorational rule $\rho_{2}$ is not the path prices would actually take under that monetary rule. Figure 6 shows, for our illustrative problem, what the actual evolution of the price level and the associated 70 percent confidence intervals are under the pseudorational policy choice. Clearly, the actual future sequence of prices would surprise the policymaker who chose $\rho_{2}$ on the basis of the pseudorational technique's projected future path.

Finally, in Figure 7 we contrast the actual evolution of the price level under the pseudorational policy $\rho_{2}$ with the actual evolution under the rational policy $\rho_{0}$. The implication is clear: a policymaker cavalierly relying on the pseudorational simulation strategy as a policy guide is likely

\footnotetext{
${ }^{17}$ See Anderson 1979, pp. 69-71.
} 
Figure 7

\section{Actual Evolution of Prices Under the Rational and Pseudorational Policies}

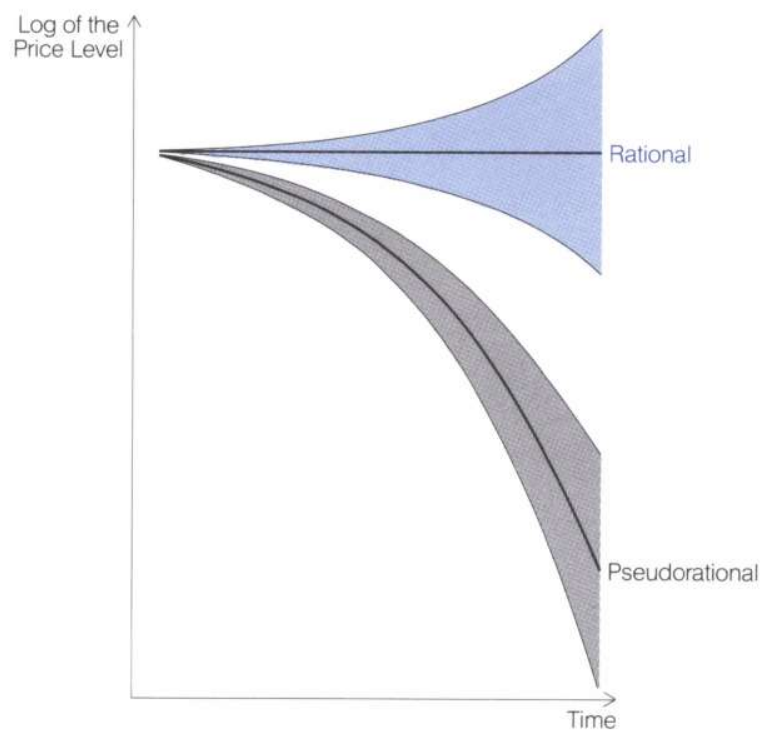

to be dramatically misled. This technique is therefore no shortcut to correctly imposing the required cross-equation restrictions on a policymaker's model.

\section{An Estimation Technique}

Still another shortcut that some investigators seem to be pursuing uses future values of the variables about which expectations are formed to estimate the model over some historical period. Alternative future scenarios are then characterized by plugging in alternative future sequences of the variable about which expectations are formed.

Although this procedure is typically applied to a policy variable like the money supply, rather than to a variable like the price level, we can use our framework to demonstrate the pitfall in this type of approach. Here the investigator characterizes (1) as

$$
m_{t}-p_{t}=-\beta\left(p_{t+1}-p_{t}\right)+w_{t}
$$

where $\beta$ is estimated over some historical period for which the sequence of one-period-ahead price levels is available.
Once the model is rearranged to give a solution for $p_{t}$, the coefficient $\beta$ can be estimated. The best estimate of that coefficient in this falsely restricted equation is $\beta=0 .{ }^{18}$ The resulting price and money relation is then

$$
p_{t}=m_{t}+\text { error }
$$

instead of the correct relation given in (3). In fact, the relation given here is correct only in the case where $\rho=1$. Thus, estimating a relation like ( $\left.1^{\prime \prime}\right)$ for econometric policy evaluation really amounts to the same thing as forecasting both by not imposing the cross-equation restrictions ( $\beta$ is not a function of $\rho$ ) and by using a wrong characterization of the monetary rule ( $\rho$ is made to equal 1 exactly).

\section{Conclusion}

Econometric models can be formulated in a way that will make them useful for policy evaluation, though the job has not been accomplished yet. The challenge is to formulate and imbed correctly in an econometric model the types of cross-equation restrictions implied by the assumption that agents form their expectations rationally. We have demonstrated in a simple case how a correctly restricted model can be a useful guide to policy choices. We have also shown why attempts to impose these restrictions on larger models should continue: methods proposed to circumvent this complicated procedure lead to incorrect policy choices and so are likely to be as counterproductive as the defective standard approaches they are intended to replace.

${ }^{18}$ The maximum likelihood estimate of $\beta$ is 0 , but it is the false restriction and not the particular estimation procedure that delivers this result. 


\section{References}

Anderson, Paul A. 1978. Rational expectations: how important for econometric policy analysis? Federal Reserve Bank of Minneapolis Quarterly Review 2 (Fall): 4-9.

1979. Rational expectations forecasts from nonrational models. Journal of Monetary Economics 5 (January): 67-80.

Cagan, Phillip. 1956. The monetary dynamics of hyperinflation. In Studies in the quantity theory of money, ed. Milton Friedman, pp. 25-117. Chicago: University of Chicago Press.

Hansen, Lars P., and Sargent, Thomas J. 1980. Formulating and estimating dynamic linear rational expectations models. Journal of Economic Dynamics and Control 2 (February): 7-46.

Lucas, Robert E., Jr. 1976. Econometric policy evaluation: a critique. In The Phillips curve and labor markets, ed. Karl Brunner and Allan H. Meltzer. Carnegie-Rochester Conference Series on Public Policy 1: 19-46. Amsterdam: North-Holland.

Lucas, Robert E., Jr., and Sargent, Thomas J. 1979. After Keynesian macroeconomics. Federal Reserve Bank of Minneapolis Quarterly Review 3 (Spring): 1-16.

Modigliani, Franco. 1978. Discussion. In After the Phillips curve: persistence of high inflation and high unemployment. Federal Reserve Bank of Boston Conference Series 19: 194-200.

Sargent, Thomas J. 1980. Rational expectations and the reconstruction of macroeconomics. Federal Reserve Bank of Minneapolis Quarterly Review 4 (Summer): $15-19$.

Sargent, Thomas J., and Wallace, Neil. 1975. Rational expectations and the theory of economic policy. Studies in Monetary Economics 2. Minneapolis: Federal Reserve Bank of Minneapolis.

Solow, Robert M. 1978. Summary and evaluation. In After the Phillips curve: persistence of high inflation and high unemployment. Federal Reserve Bank of Boston Conference Series 19: 203-209.

Supel, Thomas M. 1980. Supply-side tax cuts: will they reduce inflation? Federal Reserve Bank of Minneapolis Quarterly Review 4 (Fall): 6-15.

Swamy, P. A. V. B., and Tinsley, P. A. 1980. Linear prediction and estimation methods for regression models with stationary stochastic coefficients. Journal of Econometrics 12 (February): 103-42.

Whiteman, Charles H. 1981. Moving average representations in rational expectations models. Ph.D. dissertation, University of Minnesota. 